that all engaged in photometric research publish promptly the characteristics of their instruments, colour filters, etc., was adopted.

The report presented by the President of the Commission was accepted without alteration.

\title{
COMMISSION 26. (DOUBLE STARS.)
}

President: Prof. E. Hertzsprung.

SeCRETARY: Dr W. H. VAN DEN Bos.

The President read the draft report. No objections being entered, this was adopted by the meeting.

The President proposed an addition to the report. This was agreed to.

The adoption of a single system of orbital elements was advocated by van den Bos, who reported on the correspondence which had taken place on this subject with the members. As the result of all answers received, a system of elements was proposed and adopted by the Committee.

The President suggested that this be given in the report and van den Bos, at the request of van Biesbroeck, proposed that a full set of definitions and formulae be added. This was agreed to.

Salet asked if Committee 3 (notations) should not be consulted: van den Bos replied that all the symbols in question are already in general use and that his proposal is not a matter of notation, but of unique definition of the existing symbols.

Zagar reported for Bianchi that the $50-\mathrm{cm}$. refractor of Milan will be re-erected at Merate, to be used for visual measures in connection with photographic measures with the roo-cm. reflector.

Zagar expressed the wish for more radial velocities of visual doubles and for publication of the times of observation.

The President replied that this can be better achieved by personal correspondence.

Giacobini proposed an international distribution of the pairs to be measured over observers, measurement of neighbouring stars for deriving mass ratios and the establishment of a list of personal equation stars.

The President replied that the first two proposals are of too wide a scope to be discussed at this meeting and that for the third Giacobini's own list might be considered.

Arend reported that Uccle Observatory plans the remeasurement of the pairs observed there by van Biesbroeck in earlier years, with new optical equipment.

Commission 26 requests computers of binary star orbits to publish in future the following data, using the symbols only in the sense indicated here:

\section{Elements}

$P$ Period. In years unless otherwise stated.

$n$ Mean motion, $=360 \%$. Always positive.

$T$ Epoch of periastron passage. Not to be corrected for light-time.

e Eccentricity.

a Semi axis major of relative orbit, in seconds of arc. 
$i$ Inclination. For direct motion in the first quadrant, for retrograde motion in the second. In general two planes, making equal angles with the plane of projection, are equally possible. This is to be indicated by attaching the double sign \pm to $i$. Omit this sign after the correct plane has been established by data referring to the third coordinate.

$\Omega$ Position angle of ascending node for equinox 1900 . When $i$ carries the double sign, take $\Omega<\mathrm{I} 80^{\circ}$.

$\omega$ Longitude of periastron. Angle in plane of true orbit, counted always in the direction of motion, from the node as given to periastron.

$A, B, F, G$ The Thiele-Innes constants, which are equivalent to the classical elements $a, i, \omega, \Omega$.

$C, H, p L, p N$ For computing third coordinate and relative radial velocity. When $i$ carries the double sign, these four constants carry either \pm or $\mp$, the top sign corresponding with an ascending node under $180^{\circ}$.

A circular orbit is to be indicated by putting both $e$ and $\omega$ zero, so that $T$ indicates the time when the position angle $=\Omega$.

An orbit perpendicular to the line of sight, motion direct, is to be indicated by $i=0^{\circ}, \Omega=0^{\circ}$, so that $\omega$ is position angle of periastron; if the motion is retrograde, by $i=I 80^{\circ}, \Omega=0^{\circ}$, so that $360^{\circ}-\omega$ is position angle of periastron.

\section{Comparison of Orbit With Observations}

The Commission is of opinion that the publication of an orbit cannot be considered complete without such a comparison. Computers are therefore requested to publish the $O-C$ for the observations used or, in the case of an abundantly observed pair, for a set of normal places.

\section{EPHEMERIS}

Computers are requested to publish an ephemeris, the extent of which is left to their judgment.

For the convenience of orbit computers a set of formulae, applicable to the symbols as defined above, is given here. Union Observatory Circulars 68 and 86 may be consulted.

$$
\begin{aligned}
& e=\sin \phi \\
& X=\frac{r}{a} \cos v=\cos E-\sin \phi \\
& Y=\frac{r}{a} \sin v=\sin E \cos \phi .
\end{aligned}
$$

Tables for $X$ and $Y$ with arguments $M$ and $e$ : Appendix U.O.C. 7 I.

For ephemeris:

$$
\begin{aligned}
& x=\rho \cos \theta=A X+F Y \\
& y=\rho \sin \theta=B X+G Y \\
& z=C X+H Y .
\end{aligned}
$$

Radial velocity (companion - primary) $=L \frac{\partial X}{\partial M}+N \frac{\partial Y}{\partial M}$. 
For computing classical elements:

$$
\begin{aligned}
& \tan (\omega+\Omega)=\frac{+B-F}{+A-G} ; \quad \sin (\omega+\Omega) \text { same sign as }+B-F, \\
& \tan (\omega-\Omega)=\frac{-B-F}{+A-G} ; \quad \sin (\omega-\Omega) \text { same sign as }-B-F .
\end{aligned}
$$

In the general case take the set with $\Omega<180^{\circ}$.

or

$$
2 a=(A+G) \sec (\omega+\Omega)+(A-G) \sec (\omega-\Omega),
$$

$$
2 a=(B-F) \operatorname{cosec}(\omega+\Omega)+(-B-F) \operatorname{cosec}(\omega-\Omega) \text {, }
$$

or

$$
\begin{aligned}
\tan ^{2} \frac{i}{2} & =\frac{(A-G) \sec (\omega-\Omega)}{(A+G) \sec (\omega+\Omega)}, \\
\tan ^{2} \frac{i}{2} & =\frac{(-B-F) \operatorname{cosec}(\omega-\Omega)}{(B-\bar{F}) \operatorname{cosec}(\omega+\Omega)} .
\end{aligned}
$$

For third coordinate and relative radial velocity:

$$
\begin{aligned}
C & =a \sin \omega \sin i \\
H & =a \cos \omega \sin i \\
p L & =4.737 n C \\
p N & =4.737 n H \quad \text { (p is parallax). }
\end{aligned}
$$

These formulae give the top-signs for the general case, $i \pm$. The positive results must then be given \pm , the negative $\mp$. If the ascending node is known and $\omega$ is counted from that, the formulae give the correct signs.

For computing the Thiele-Innes constants from the classical elements:

$$
\begin{array}{lll}
A^{\prime}=a \cos \omega \cos \Omega & A^{\prime \prime}=G^{\prime} \cos i & A=A^{\prime}-A^{\prime \prime} \\
B^{\prime}=a \cos \omega \sin \Omega & B^{\prime \prime}=F^{\prime} \cos i & B=B^{\prime}+B^{\prime \prime} \\
F^{\prime}=a \sin \omega \cos \Omega & F^{\prime \prime}=B^{\prime} \cos i & F=-F^{\prime}-F^{\prime \prime} \\
G^{\prime}=a \sin \omega \sin \Omega & G^{\prime \prime}=A^{\prime} \cos i & G=-G^{\prime}+G^{\prime \prime},
\end{array}
$$

or from

$$
\begin{aligned}
A+G & =a \cos (\omega+\Omega) \cdot(\mathrm{I}+\cos i) \\
A-G & =a \cos (\omega-\Omega) \cdot(\mathrm{I}-\cos i) \\
B-F & =a \sin (\omega+\Omega) \cdot(\mathrm{I}+\cos i) \\
-B-F & =a \sin (\omega-\Omega) \cdot(\mathrm{I}-\cos i) .
\end{aligned}
$$

For deriving $C, H, a$ and $i$ directly from Thiele-Innes constants:

$$
\begin{array}{ccc}
2 k=A^{2}+B^{2}+F^{2}+G^{2} & 2 l=A^{2}+B^{2}-F^{2}-G^{2} \quad m=A G-B F \quad j^{2}=k^{2}-m^{2} \\
C^{2}=j-l \quad H^{2}=j+l \quad a^{2}=j+k \quad \cos i=\frac{m}{a^{2}} .
\end{array}
$$


For differential corrections:

Weights $W_{x}$ and $W_{y}$ in $x$ and $y$ from $W_{\rho}$ and $W_{\theta}$ in distance and arc

$$
\begin{gathered}
\frac{I}{W_{x}}=\frac{\sin ^{2} \theta}{W_{\theta}}+\frac{\cos ^{2} \theta}{W_{\rho}} \quad \frac{I}{W_{y}}=\frac{\cos ^{2} \theta}{W_{\theta}}+\frac{\sin ^{2} \theta}{W_{\rho}}, \\
\Delta x=X \Delta A+Y \Delta F+P_{x} \Delta e-Q_{x} n \Delta T+R_{x} \Delta n \\
\Delta y=X \Delta B+Y \Delta G+P_{y} \Delta e-Q_{y} n \Delta T+R_{y} \Delta n \\
P_{x}=A \frac{\partial X}{\partial e}+F \frac{\partial Y}{\partial e} \quad Q_{x}=A \frac{\partial X}{\partial M}+F \frac{\partial Y}{\partial M} \quad R_{x}=Q_{x}(t-T) \\
P_{y}=B \frac{\partial X}{\partial e}+G \frac{\partial Y}{\partial e} \quad Q_{y}=B \frac{\partial X}{\partial M}+G \frac{\partial Y}{\partial M} \quad R_{y}=Q_{y}(t-T) \\
\frac{\partial X}{\partial e}=-0.0 I\left[I+\frac{Y^{2}}{\cos ^{2} \phi\left(\cos ^{2} \phi-X \sin \phi\right)}\right], \text { always negative. } \\
\frac{\partial Y}{\partial e}=+0.0 \mathrm{I} \frac{X Y}{\cos ^{2} \phi\left(\cos ^{2} \phi-X \sin \phi\right)}, \text { positive when } X \text { and } Y \text { same sign. } \\
\frac{\partial X}{\partial M}=-0.017453 \frac{Y}{\cos \phi\left(\cos ^{2} \phi-X \sin \phi\right)}, \text { opposite sign to } Y . \\
\frac{\partial Y}{\partial M}=+0.017453 \frac{\cos \phi(X+\sin \phi)}{\cos ^{2} \phi-X \sin \phi}, \text { negative when } X+e \text { negative. }
\end{gathered}
$$

These four partial derivatives can be read from the $X Y$ tables, as the mean of the preceding and the following first differences is a sufficient approximation to the first derivative. The above rules afford an easy check on the signs. The solution gives $\Delta A, \Delta B, \Delta F, \Delta G$ in seconds of arc, $\Delta e$ in units of the second decimal, $\Delta T$ in years and $\Delta n$ in degrees per annum.

Checks:

$$
A F+B G+C H=0 \quad A^{2}+B^{2}+C^{2}=F^{2}+G^{2}+H^{2}=a^{2} .
$$

Differential relations:

$$
\begin{aligned}
& \Delta A=A \frac{\Delta a}{a}+F \Delta \omega-B \Delta \Omega+C \sin \Omega \Delta i \\
& \Delta B=B \frac{\Delta a}{a}+G \Delta \omega+A \Delta \Omega-C \cos \Omega \Delta i \\
& \Delta F=F \frac{\Delta a}{a}-A \Delta \omega-G \Delta \Omega+H \sin \Omega \Delta i \\
& \Delta G=G \frac{\Delta a}{a}-B \Delta \omega+F \Delta \Omega-H \cos \Omega \Delta i \\
& \Delta C=C \frac{\Delta a}{a}+H \Delta \omega \quad+C \cot i \Delta i \\
& \Delta H=H \frac{\Delta a}{a}-C \Delta \omega \quad+H \cot i \Delta i,
\end{aligned}
$$


and the other way

$$
\begin{aligned}
& a \Delta a=\quad+A^{\prime} \Delta A \quad+B^{\prime} \Delta B \quad-F^{\prime} \Delta F \quad-G^{\prime} \Delta G \\
& a^{2} \sin i \Delta i=+\left(G^{\prime}+G^{\prime \prime}\right) \Delta A+\left(F^{\prime}-F^{\prime \prime}\right) \Delta B+\left(B^{\prime}-B^{\prime \prime}\right) \Delta F-\left(A^{\prime}+A^{\prime \prime}\right) \Delta G \\
& -a^{2} \sin ^{2} i \Delta \omega=+\left(F^{\prime}-F^{\prime \prime}\right) \Delta A+\left(G^{\prime}+G^{\prime \prime}\right) \Delta B+\left(A^{\prime}+A^{\prime \prime}\right) \Delta F+\left(B^{\prime}-B^{\prime \prime}\right) \Delta G \\
& a^{2} \sin ^{2} i \Delta \Omega=-\left(B^{\prime}-B^{\prime \prime}\right) \Delta A+\left(A^{\prime}+A^{\prime \prime}\right) \Delta B+\left(G^{\prime}+G^{\prime \prime}\right) \Delta F-\left(F^{\prime}-F^{\prime \prime}\right) \Delta G \\
& C \Delta C=\begin{array}{llll}
+A^{\prime \prime} \Delta A & -B^{\prime \prime} \Delta B & -F^{\prime} \Delta F & -G^{\prime} \Delta G
\end{array} \\
& H \Delta H=\quad+A^{\prime} \Delta A \quad+B^{\prime} \Delta B \quad+F^{\prime \prime} \Delta F \quad-G^{\prime \prime} \Delta G .
\end{aligned}
$$

In these formulae $\Delta i, \Delta \omega, \Delta \Omega$ are in radians, the others in seconds of arc.

\section{COMMISSION 27. (VARIABLE STARS.)}

\section{President: Dr A. A. Nijland. \\ SECRETARY: Mr FELIX DE ROY.}

The draft report was discussed, and adopted with some alterations and additions.

The following resolutions were referred to the General Assembly:

I. The Commission recommends that the yearly subsidy granted by the Union for the publication of the Cracow Ephemerides of Eclipsing Binaries (Prof. T. Banachiewicz) be continued to the amount of 700 gold francs, and expresses the wish that the decimal division of the day used in these ephemerides will be, in the future, counted from Greenwich Noon.

2. The Union having endorsed the proposal made by Prof. Grouiller to compile a list of unpublished observations of Variable Stars, and recommended that this compilation be published by the Union, the Commission recommends that a subsidy of 200 gold francs should be granted to that object.

3. The Commission recommends that Prof. Nijland's Lists $A, B$, and $C$, brought up to date, together with a new list $D$, should be published by the Union with a subsidy not exceeding 300 gold francs.

In addition, the following recommendations were adopted:

4. The Commission expresses the wish that all observations of Variable Stars (including Novae) should be recorded in Greenwich Mean Astronomical Time, counted from noon to noon, and directs the attention of editors of astronomical publications towards this point.

5. The Commission recommends the simultaneous observation, by photometric and spectrographic methods, of eclipsing binaries, and suggests that, as far as practicable, interested astronomers should coordinate their efforts to bring about this result.

6. The Commission recommends that a selected list (see p. I72 of the Report) of six Long Period Variables be observed by experienced observers through Schott's VGI, $2 \mathrm{~mm}$. green filter, and expresses the wish that a photographic and a photovisual sequence of comparison stars for these stars be measured and published as soon as possible.

7. The Commission recommends that, with a view to studying the secondary variations in the period of eclipsing binaries, at least one epoch of minimum should 